\title{
SOME REMARKS ON CERTAIN CLASSES OF SEMILATTICES
}

\section{P.V. RAMANA MURTY and $M$. KRISHNA MURTY}

Department of Mathematics

Andhra University

Waltair 530003

India.

(received June 28, 1979 and in revised form March 7, 1980)

ABSTRACT. In this paper the concept of a $*$-semilattice is introduced as a generalization to distributive *-lattice first introduced by Speed [1]. It is shown that almost all the results of Speed can be extended to a more general class

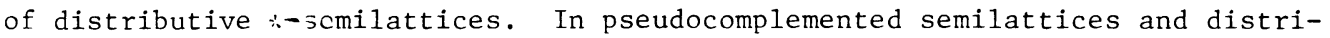
butive semilattices the set of annihilators of an element is an ideal in the sense of Grätzer [2]. But it is not so in general and thus we are led to the definition of a weakly distributive semilattice. In $\S 2$ we actually obtain the interesting corollary that a modular $\dot{x}$-semilattice is weakly distributive if and only if its dense filter is neutral. In $\S 3$ the concept of a sectionally pseudocomplemented semilattice is introduced in a natural way. It is proved that given a sectionally pseudocomplemented semilattice there is a smallest quotient of it which is a sectionally Boolean algebra. Further as a corollary to one of the theorems it is obtained that a sectionally pseudocomplemented semilattice with a dense element becomes a $*$-semilattice. Finally a necessary and sufficient condition for a *-semilattice to be a pseudocomplemented semilattice is obtained. 
KEY WORDS AND. PHRASES. Pseudocomplemented semilattice, :-semilattice, weakly distributive semilatice, Neutral filter, Sectionally pseudocomplemented semilattice.

\section{MATHEMATICS SUBJECT CLASSIFICATION CODES: 06}

1. INTRODUCTION.

Speed [1] has introduced the concept of a distributive $*-1$ attice. In this paper we observe that the condition he has placed on a distributive lattice to become a distributive *-lattice can be placed on any meet semilattice with 0 and accordingly in this paper we call such semilatticesas *-semilattices (see definition 1). It is shown that almost all the results of Speed can be extended to a more general class of distributive -semilattices. The concept of a filter in join semilattice and hence (dually) that of an ideai in a meet semilattice is due to Grätzer [2]. In pseudocomplemented semilattices and distributive semilattices the set of annihilators of an element is an ideal in the sense of Grätzer. But it is not so in general and thus we are led to the definition of a weakly distributive semilattice. In $\$ 2$ we prove as a corollary to theorem 4 that a modular $\dot{*}$-semilattice is weakly distributive if and only if its dense filter is neutral. Further we show in Theorem 3 that the filter congruence induced by the dense filter on a modular $*$-semilattice $\mathrm{L}$ is equal to the well known congruence $R$ on $L$ defined by $R=\left\{(x, y) \in L x L \mid(x)^{*}=(y)^{*}\right\}$.

In $\S 3$ we study sectionally pseudocomplemented semilattices the concept of which is introduced in [3] and [4]. Actually we prove in Theorem 8 that given a sectionally pseudocomplemented semilattice there is a smallest quotient of it which is a sectionally Boolean algebra. Further we show in Theorem 10 that given a sectionally pseudocomplemented semilattice $\mathrm{L}$ there is a Boolean algebra $\mathrm{S}$ such that if $L$ has a dense element then $L / R$ is a Boolean algebra isomorphic to $S$, and obtain as a corollary that a sectionally pseudocomplemented semilattice with a dense element becomes a $*-\xi$ mmilattice. Finally we obtain a necessary and sufficient condition for a $*$-semilattice to be a pseudocomplemented semilattice.

2. RESULTS ON MODULAR *-SEMILATTICES. 
DEFINITION 1. Let $\mathrm{S}$ be a meet semilattice with 0 ; then for any subset $\mathrm{A}$ of $S, A^{*}$ stands for $\{x \in S \mid x \wedge a=0$ for all $a \in A\}$. If $A=\{a\}$ then we denote $A^{*}$ by $(a)^{*}$. An element $a \in S$ is called a dense element of $S$ if and only if $(a) *=\{0\}$. $D$ denotes the set of all dense elements of $S$ and it can be seen that $D$ is a filter of $S$ (if $D$ is nonempty).

The concept of a distributive $*-1$ attice is due to speed [1]. However the condition he has placed on a distributive lattice in his definition may as we11 be placed on any meet semilattice with 0 as in the following:

DEFINITION 2. A meet semilattice with 0 is said to be a $*$-semilattice if and only if for any $a \in L$ there exists $a^{\prime} \in L$ such that $(a)^{*}=\left(a^{\prime}\right)^{* *}$.

DEFINITION 3. (Gratzer [2] and Katriňak [5]). A nonempty subset I of a meet semilattice $\mathrm{S}$ is called an ideal of $\mathrm{S}$ if and only if

(i) $x \in I$ and $a \leq x(a \in S)$ implies $a \in I$

(ii) $x, y \in I$ implies the existence of $z$ in $I$ such that $y \leq z$ and $y \leq z$.

DEFINITION 4. An ideal I of a meet semilattice $S$ is said to be a prime ideal if and only if $a \wedge b \in I(a, b \in S)$ implies either $a$ or $b \in I$.

DEFINITION 5. A meet semilattice $(S, \wedge)$ is called a distributive semilattice if and only if $x, y, z \in S$ and $x \geq y \wedge z$ imply the existence of $y_{1}, z_{1} \in S$ such that $\mathrm{y}_{1} \geq \mathrm{y}, \mathrm{z}_{1 \geq \mathrm{z}}$ and $\mathrm{x}=\mathrm{y}_{1} \wedge \mathrm{z}_{1}$.

DEFINITION 6. A meet semilattice $L$ with 0 is called a weakly distributive semilattice if $(a)^{*}$ is an ideal for every $a \in L$. The notion of weak distibutivity in semilattices is a natural generalization of the notion of 0 -distributivity introduced by Varlet [6]. Clearly every pseudocomplemented semilattice and every distributive meet semilattice with 0 is weakly distribitive. Also one can onserve that in a weakly distributive semilattice $S$, a proper filter $U$ is an ultra filter of $\mathrm{S}$ if and only if $\mathrm{S}-\mathrm{U}$ is a minimal prime ideal of $\mathrm{S}$.

It will be shown in this article that almost all the results of speed [1] for distributive *-lattices (see Theorems 1,2 and 6 of this paper) can be extended to weakly distributive $*$-semilattices and proofs are given only for those results which can not be obtained from those of Speed. 
From now onwards in this article, L denotes a weakly distributive semilattice. Let $M$ be the set of all minimal prime ideals of L. For any subset A of L write $h(A)=\{M \in M \mid A \subseteq M\}$ and $M(A)=\{M \in M \mid A \ddagger M\}$. Then $\{M(A) \mid A \subseteq L\}$ of subsets of $M$ is a topology on $M$ called the hull-kernel topology and $\{M\{\mathbf{x}\}=M \mid \mathbf{x} \in L\}$ is a base for the above topology. Also $\{h(a) \mid a \in I\}$ is a base for some topology on $M$ called the dual hull-kernel topology.

LEMMA 1. In $\mathrm{L}$ the following hold:

(i) A prime ideal $M$ is minimal if and only if $(x)^{*}-M \neq \emptyset$ for every $x \in M$

(ii) $\quad M_{\mathrm{x}}=\mathrm{h}\left((\mathrm{x})^{\star}\right)$

(iii) $h(x)=h\left((x)^{* *}\right)$

(iv) $(z)^{*}=(x)^{*} \Gamma_{1}(y)^{*} \Longleftrightarrow h(z)=h(x) r_{1} h(y)$

(v) $(x \wedge y)^{* *}=(x)^{* *} \Gamma_{1}(y)^{* *}$

(vi) $\quad(\mathrm{x})^{* *}=(\mathrm{y})^{*} \Leftrightarrow \mathrm{h}(\mathrm{x})=\mathrm{h}\left((\mathrm{y})^{*}\right)$

If we write $T_{h}$ for the hull-kernel topology and $T_{d}$ for the dual hull-kerneltopology on $M$ then we have the Theorem:

THEOREM 1. In $\mathrm{L}$, the following conditions are equivalent.

(1) L is a + -semilattice

(2) $T_{h}=T_{d}$.

(3) $M$ is compact in the hull-kernel topology.

PROOF. $\quad(1) \Rightarrow(2)$ and $(2) \Rightarrow(3)$ are straightforward. $\quad(3) \Rightarrow(1)$ : Assume that $M$ is compact. Now $h(x)=N^{\prime} x$ is a closed subset of Mand hence is a compact subset of $M$. Also $\Gamma_{(}\left(h(x) r_{h}(t)\right)=\emptyset$ so that by compactness of $h(x)$ it follows $t \in(x)^{*}$ that $h(x) / h\left(t_{1}\right) \gamma_{1} \ldots\left(, h\left(t_{n}\right)=\emptyset\right.$ for some $t_{1}, \ldots, t_{n} \in(x) *$ and hence there exists $z \in(x)$ * such that $t_{i \leq z}$ for $1 \leq i \leq n$. Now $h((x))^{*}=h(z)$ and hence $(x)^{*}=(z)^{* *}$. Thus $(3) \rightrightarrows(1)$. Q.E.D. THEOREM 2. In $\mathrm{L}$ the following are equivalent.

(1) L is a *-semilattice.

(2) For any $x \in L$ there exists $x^{\prime} \in L$ such that $x \wedge x^{\prime}=0$ and $[x)\left(,\left[x^{\prime}\right) \subseteq D\right.$ 
PROOF. (1) $\Rightarrow(2)$ is obvious. Assume (2). Clearly $\left(x^{\prime}\right) * \star \subseteq(x) *$. If $t \wedge x$ $=0$ and $r \wedge x^{\prime}=0$ then $x, x^{\prime} \in(t \wedge r) *$ so that there exists $z \geq x, x^{\prime}$ and $z \wedge t \wedge$ $r=0$ and hence $z \in D$. Thus $t \wedge r=0$.

If $(S, \wedge)$ is a meet semilattice and $F$ is a filter of $S$ then $\theta_{F}=\{(x, y) \mid x \wedge f$ $=\mathrm{y} \wedge \mathrm{f}$ for some $\mathrm{f} \in \mathrm{F}\}$ is a congruence on $\mathrm{S}$ called the congruence induced by the filter F. Q.E.D.

DEFINITION 7. (see Rhodes [7]). A meet semilattice $(S, \wedge)$ is called a modular semilattice if and only if $x, y, z \in S$ and $x \geq y \wedge z$ imply the existence of $y_{1}, z_{1} \in S$ such that $x \wedge y_{1}=x \wedge z_{1}=y_{1} \wedge z_{1}$.

THEOREM 3. In a modular $*$-semilattice $S$ we have $\phi=R$ where $R=\{(x, y)$ $\left.(x)^{*}=(y)^{*}\right\}$.

PROOF. Clearly $\theta_{\mathrm{D}} \subseteq \mathrm{R}$. Now let $(\mathrm{x})^{*}=(\mathrm{y})^{*}$. Evidently $(\mathrm{x} \wedge \mathrm{y})^{*}=(\mathrm{x})^{*}$. By modularity of $\mathrm{S}, \mathrm{y} \wedge \mathrm{x}^{\prime}=0 \leq \mathrm{y} \wedge \mathrm{x} \leq \mathrm{y}$ implies $\mathrm{y} \wedge \mathrm{x}=\mathrm{y} \wedge \mathrm{x}_{2}$ for some $\mathrm{x}_{2} \geq \mathrm{x}^{\prime}$. By Theorem 2, $x_{2} \geq x^{\prime}$ and $x_{2} \geq y \wedge x$ imply $x_{2} \in D$. Similarly $x \wedge x^{\prime}=0 \leq y \wedge x \leq x$ implies $y \wedge x=y_{2} \wedge x$ for some $y_{2} \geq x^{\prime}$. As also $y_{2} \in D$, we have $(x, y) \in \theta_{D}$ since $\mathrm{x} \wedge \mathrm{x}_{2} \wedge \mathrm{y}_{2}=\mathrm{y} \wedge \mathrm{x}_{2} \wedge \mathrm{y}_{2}$ and thus $\theta_{\mathrm{D}}=\mathrm{R}$

Q.E.D.

It can be observed that if $S$ is not modular then $\theta$ need not be equal to $R$ even if $\mathrm{S}$ is pseudocomplemented. In the following theorem a necessary and sufficient condition for a modular *-semilattice to be weakly distributive is obtained.

THEOREM 4. If $\mathrm{S}$ is $\mathrm{a} *$-semilattice which is directed above such that $\theta_{D}=R$ then $S$ is a weakly distributive semilattice if and only if for all $x, y \in S$

$$
([\mathrm{x}) \cap[\mathrm{y})) \vee \mathrm{D}=([\mathrm{x}) \vee \mathrm{D}) \cap([\mathrm{y}) \vee \mathrm{D})
$$

PROOF. Assume that $S$ is weakly distributive. Let $t \in S$ and $l e t x \wedge d \leq t$ and $y \wedge d \leq t$ for some $d \in D$. Then there exists $z \in S$ such that $x \leq z, y \leq z$ and $z \wedge t$ ' $=0$. Now we have $(z) *=(z \wedge t) *$ so that there exists $e \in D$ such that $z \wedge e \leq t$ and hence $([\mathrm{x}) \vee \mathrm{D}) \cap([\mathrm{y}) \vee \mathrm{D}) \subseteq([\mathrm{x}) \cap[\mathrm{y})) \vee \mathrm{D}$. Conversely suppose (I) holds and $x, y \in(a) *$ so that $x \wedge a=y \wedge a=0$ which implies that $(x) * *$ and $(y) * * \subseteq(a) *$ $=\left(a^{\prime}\right) * *$. Thus we have $\left(x \wedge a^{\prime}\right) *=(x) *$ and $(y \wedge a) *=(y) *$. Therefore, $x \wedge a^{\prime}$ $[x) \vee D$ and $y \wedge a^{\prime} \in[y) \vee D$. Hence, using $\theta_{D}=R, a^{\prime} \in([x) \vee D) \cap([y) \vee D$.

By (I) there exists $e \in D$ such that $z \wedge e \leq a^{\prime}$ for some $z \geq x, y$. 
Since $z \wedge e \wedge a \leq a \wedge a^{\prime}=0$, we have $z \wedge a=0$ and so $z \in(a) *$.

Q.E.D.

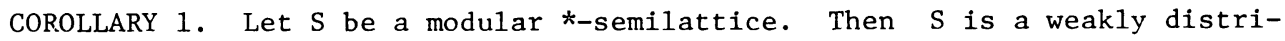
butive semilattice if and only if $D$ is neutral in the lattice of filters of $S$.

THEOREM 5. Let $\mathrm{L}$ be a meet semilattice with 0 and $\mathrm{D} \neq \emptyset$, then the following are equivalent.

(1) $\mathrm{L} / \theta$ is pseudocomplemented; (2) L is a $*$-semilattice satisfying the condition $x \wedge z=0$ implies the existence of $d \in D$ such that $x \wedge d \leq z^{\prime}$ where $(z) * *=\left(z^{\prime}\right) *$. PROOF. $\quad(1) \Rightarrow(2)$ : Let $x \in L$ and let $\left(\theta_{D}(x)\right) *=\theta_{D}(y)$. We now claim that $(\mathrm{x}) *=(\mathrm{y}) * *$. Since $\mathrm{x} \wedge \mathrm{y}=0$ we have $(\mathrm{y}) * * \subseteq(\mathrm{x}) *$. Suppose $\mathrm{r} \wedge \mathrm{x}=0$ and $t \wedge y=0$ so that there exists $d \in D$ such that $r \wedge y \wedge d=r \wedge d$ and hence $r \wedge t \wedge$ $\mathrm{d}=0$. Therefore $\mathrm{r} \wedge \mathrm{t}=0$ and thus $\mathrm{r} \in(\mathrm{y}) * *$ and hence $(\mathrm{x}) *=(\mathrm{y}) * *$. Thus $\mathrm{L}$ is a *-semilattice. Now if $x \wedge z=0$ then $\theta_{D}(x) \leq \theta_{D}(z) *=\theta_{D}\left(z^{\prime}\right)$ where $(z) * *=\left(z^{\prime}\right) *$. Clearly $(2) \Rightarrow(1)$.

Q.E.D.

COROLLARY 2. If $\mathrm{L}$ is a modular similattice with 0 and $\mathrm{D} \neq \emptyset$ then $\mathrm{L}$ is a *-semilattice if and only if $\mathrm{L} / \mathrm{Q}$ is pseudocomplemented.

PROOF. By Theorem 3 and the above Theorem 5 . Q.E.D.

Theorem 2 of $\$ 5$ in [1] can as well be generalized to modular $*$-semilattices as in the following:

THEOREM 6. If $\mathrm{L}$ is a modular *-semilattice, then the following are equiva1ent:

(1) L is a Boolean algebra

(2) L is a disjunctive semilattice, that is, $a, b \in L$ and $a<b$ implies the existence of $c \in L$ such that $a \wedge c=0$ and $b \wedge c \neq 0$.

(3) 1 is the only dense element of L.

PROOF. Clearly (1) $\Rightarrow(2)$ and $(2) \Rightarrow(3)$. Now assume (3). By (3) $\theta_{\mathrm{D}}=\mathrm{id}$. Therefore $\mathrm{L} / \theta_{\mathrm{D}} \cong \mathrm{L}$ and by Corollary $2, \mathrm{~L}$ is pseudocomplemented. Since $\mathrm{L}$ is a modular *-semilattice, $D \neq \emptyset$. Q.E.D.

\section{SECTIONALLY PSEUDOCOMPLEMENTED SEMILATTICES.}

In this article a further study is made of the concept of a sectionally 
Pseudocomplemented semilattice introduced by Katrinák [3] and [4]. DEFINITION 8 (see [3] and [4]). A meet semilattice L with 0 is called a sectionally pseudocomplemented semilattice if and only if for every a $\in I$, the interval $[0, a]$ is a pseudocomplemented semilattice.

Everv pseudocomplememnted semilattice is a sectionally pseudocomplemented semilattice where for $a \in L$ and $x \in[0, a]$ the pseudocomplement of $x$ in this interval $[0, a]$ is given by $x^{*} \wedge$ a.

Throughout this article L stands for a sectionally pseudocomplemented semilattice and for any $x \in[0, a]$ the pseudocomplement of $x$ in $[0, a]$ is denoted by $\mathrm{x}^{\circ} \mathrm{a}$

LFMMA 2. For any $x, y, z \in L$ we have

$$
(x \wedge y \wedge z)^{*} y \wedge z=y \wedge(x \wedge z)^{*} z=z \wedge(x \wedge y){ }^{*} y
$$

PROOF. Since $y \wedge(x \wedge z)^{*} z \leq y \wedge z$ and $y \wedge(x \wedge z)^{*} z \wedge x \wedge y \wedge z=0$ We have $y \wedge(x \wedge z)^{*} z \leq(x \wedge y \wedge z)^{*} y \wedge z$. Now $(x \wedge y \wedge z)^{*} y \wedge z \leq y, z$ and $(x \wedge y \wedge z)^{*} y \wedge z \wedge x \wedge z=0$ and hence $(x \wedge y \wedge z)^{* y \wedge z} \leq y \wedge(x \wedge z)^{*}$.

DEFINITION 9. A meet congruence $\emptyset$ on $L$ is said to be a $\star$-congruence if and only if $\left((x \wedge a)^{*} a,(y \wedge a)^{*} a\right) \in \emptyset$ for all a $\in$ L whenever $(x, y) \in \emptyset$.

THEOREM 7. If $\emptyset$ is a $*$-congruence on $L$, the $L / \varnothing$ is a sectionally pseudocomplemented semilattice where $(\emptyset(x))^{*} \emptyset(a)=\emptyset\left((x \wedge a){ }^{*} a\right)$.

PROOF. Suppose $\emptyset(\mathrm{x}) \leq \emptyset(\mathrm{a})$. Clearly $\emptyset(\mathrm{x}) \wedge \emptyset\left((\mathrm{x} \wedge \mathrm{a})^{*} \mathrm{a}\right)=\emptyset(0)$. Suppose $\emptyset(y) \leq \emptyset(a)$ such that $\emptyset(x) \wedge \emptyset(y)=\emptyset(0)$ then $(x \wedge y \wedge a, 0) \in \emptyset$ so that $\left((x \wedge y \wedge a){ }^{*} y \wedge a\right.$, $y \wedge a) \epsilon \emptyset$ and hence by the above lemma $\left(y \wedge(x \wedge a)^{*} a, y \wedge a\right) \in \emptyset$. Therefore $\emptyset(y)=\emptyset(y \wedge a) \leq \emptyset\left((x \wedge a)^{*} a\right) \quad$ O.E.D.

DEFINITION 10. If $\mathrm{L}_{1}$ and $\mathrm{L}_{2}$ are sectionally pseudocomplemented semilattices, then a meet homomorphism $\mathrm{f}: \mathrm{L}_{1} \rightarrow \mathrm{L}_{2}$ is said to be a $*$-homomorphism if and only if $f\left(x^{*} \mathrm{a}\right)=(\mathrm{f}(\mathrm{x}))^{*} \mathrm{f}(\mathrm{a})$ for $\mathrm{all} \mathrm{x}, \mathrm{a} \in \mathrm{L}$, and $\mathrm{x} \leq \mathrm{a}$. Note that if $\mathrm{f}: \mathrm{L}_{1} \rightarrow \mathrm{L}_{2}$ is a *-homomorphism then kernel $\mathrm{f}$ is a *-congruence and every filter congruence on $\mathrm{L}$ is a *-congruence.

DEFINITION 11. If in L every section $[0, a]$ is a Boolean algebra for all $a \in L$ then we call $\mathrm{L}$ as a sectionally Boolean algebra, also called as a (dual) 
semi-Boolean algebra in the sense of Abbott [8].

In the following theorem we now show that given a sectionally pseudocomplemented semilattice there is a smallest quotient of it which is a sectionally Boolean algebra.

THEOREM 8 . If we define $\theta$ on $\mathrm{L}$ by $(x, y) \in \theta$ if and only if $(x \wedge a)^{*} a=(y \wedge a)$ for all a $\in \mathrm{L}$, then $\theta$ is a $*$-congruence on $\mathrm{L}$ such that $\mathrm{L} / \theta$ is a sectionally Boolean algebra and if for any *-congruence $\emptyset$ on $\mathrm{L}, \mathrm{L} / \emptyset$ is a sectionally Boolean algebra then $\theta \subseteq \emptyset$.

PROOF. First observe that $\theta=R$ (see theorem 3, §2). Also observe for any $*$-congruence $\emptyset$ on $\mathrm{L}$

$$
(\emptyset(x \wedge a))^{*} \emptyset(a)=\emptyset\left((x \wedge a)^{* a}\right) .
$$

If $a \in L$ then $S_{a}$ denotes the Boolean algebra of closed elements of $[0, a]$. THEOREM 9. If we write $S=\left\{x \in \underset{a}{\pi} \epsilon_{L} S \mid x(a)=x(b) \wedge\right.$ a whenever $\left.a \leq b\right\}$, then $S$ is a Boolean algebra and there is a *homomorphism $f: L \rightarrow S$ (whose kernel is $\theta$ ) such that for each $a \in L$ we have $S_{a}$ and $[0, f(a)]$ are isomorphic.

PROOF. It can be seen that $S$ is in fact a subalgebra of $\pi_{a} \epsilon_{L} S_{a}$. Now define $f: L \rightarrow S$ by $f(x)(a)=(x \wedge a)^{* * a}$. Let $x \in L$ and $a \in L$ with $x \leq a$. If $b \in L$ then $f\left(x^{* a}\right)(b)=f\left((x \wedge a)^{* a}\right)(b){ }_{*}($ since $x \leq a)=\left((x \wedge a)^{* a} \wedge b\right)^{* * b}=\left((x \wedge a \wedge b)^{* a} \wedge b\right)=$ $\left((x \wedge b){ }^{*} b \wedge a \wedge b\right){ }^{* *} b=(x \wedge b)^{*} b \wedge(a \wedge b)^{* * b}=(f(x)(b))^{*} b \wedge f(a)(b)=\left((f(x))^{\prime} \wedge\right.$ $f(a))(b)=(f(x)){ }^{*} f(a)(b)$. Thus $f$ is $a *$-homomorphism and ker $f=\theta$. It can be seen that the map $x \mapsto f(x)$ is a homomorphism of $S_{a}$ into $[0, f(a)]$. Clearly kernel of the above map is $\{0\}$ so that the map is a monomorphism. Now we shall prove that it is onto. Let $y \in S$ such that $y \leq f(a)$. We claim that $f(y(a))=y$. If $b \in L$, then observe that $y(a) \wedge b=y(b \wedge a)=y(b) \wedge a \cdot \quad C l e a r 1 y(y(b) \wedge a)^{* *} b$ $\leq y(b)$. Now $y(b) \leq y(b) \wedge f(a)(b)=y(b) \wedge(a \wedge b)^{* *} b=(y(b) \wedge a \wedge b)^{* *} b$ $=(y(b) \wedge a)^{* *_{b}}$. Thus the map is an isomorphism. O.E.D. REMARK. If $\mathrm{L}$ is also directed above then it can be seen that $\mathrm{S}$ is the inverse limit of $\left\{s_{a}, \emptyset_{a b}, L\right\}$ where $\emptyset_{a b}: s_{b} \rightarrow s_{a}(a \leq b)$ defined by $\emptyset_{a b}(x)=x \wedge a$. THEOREM 10. If $\mathrm{L}$ has a dense element then $\mathrm{L} / \theta$ is a Boolean algebra isomorphic to $\mathrm{S}$. 
PROOF. Let $d \in L$ be dense so that $L / \theta \cong S_{d}$ under the map $\theta[x] \mapsto(x \wedge d)^{* *} d$ and by theorem 9 we have $s_{d} \cong[0, f(d)]$. Since $f(d)(b)=b$ for all $b$ we have $[0, f(d)]=S$ so that $L / \theta \cong S$.

Q.E.D.

COROLLARY 3. If $\mathrm{L}$ has a dense element then $\mathrm{L}$ is a $\star$-semilattice.

PROOF. Follows from Theorem 1 in [9].

Finally it is natural to ask when does a *-semilattice become a sectionally pseudocomplemented semilattice. Since every section of a $*$-semilattice is also a $*$-semilattice we will prove the following:

THEOREM 11. A necessary and sufficient condition for a $x$-semilattice $S$ to be pseudocomplemented is that (1) for each $x \in S$ there exists a greatest $x^{\prime} \in S$ such that $(x)^{*}=(x)^{* *}$ and $(2) x \leq y$ implies $x^{\prime \prime} \leq y^{\prime \prime}$.

PROOF. If $\mathrm{S}$ is a pseudecomplemented semilattice, then taking the pseudocomplement of $x$ as $x^{\prime}$ it can be seen that (1) and (2) are satisfied. Conversely suppose that $\mathrm{S}$ is a $*$-semilattice satisfying (1) and (2). First observe that for any $x, y \in S$ we have $(x)^{*}=(y)^{*}$ if and only if $x^{\prime}=y^{\prime}$. Since $\left(x^{\prime}\right)^{*}=(x)^{* *}$ we have $x \leq x^{\prime \prime}$ and since $(x)^{*}=\left(x^{\prime}\right)^{*}$ we have $x^{\prime}=x^{\prime}{ }^{\prime}$. Suppose $x \wedge y=0$. Then $\left(x \wedge y^{\prime}\right)^{*}=(x)^{*}$ and hence $\left(x \wedge y^{\prime}\right)^{\prime \prime}=x^{\prime \prime}$. Now we claim that $\left(x \wedge y^{\prime}\right)^{\prime \prime}$ $=x^{\prime \prime} \wedge y^{\prime}$. Since $\left(\left(x \wedge y^{\prime}\right)^{\prime \prime}\right)^{*}=\left(x \wedge y^{\prime}\right)^{*}=\left(x^{\prime \prime} \wedge y^{\prime}\right)^{*}$ we have $x^{\prime \prime} \wedge y^{\prime} \leq\left(x \wedge y^{\prime}\right)^{\prime \prime}$. Now $\left(x \wedge y^{\prime}\right)^{\prime \prime} \leq x^{\prime \prime} \wedge y^{\prime}\left(\right.$ by (2)). Hence $x^{\prime \prime} \wedge y^{\prime}=x^{\prime \prime}$ so that $x \leq x^{\prime \prime} \leq y^{\prime}$ and hence $\mathrm{S}$ is pseudocomplemented. O.E.D.

\section{ACKNOWLEDGEMENT :}

We thank the referee for his valuable suggestions and comments which helped in shaping the paper into the present form. 


\section{REFERENCES}

1. SPEED, T.P. Some Remarks on a class of Distributive lattices, J. Aust. Math. Soc., IX (1969), 289-296.

2. GRÄTZER, G. Lattice Theory. First Concepts and Distributive lattices, W.H. Freeman and Company, 1971.

3. KATRIÑ́́K, T. Notes on Stone lattices I, Mat. fyz. Casop, SAV 16 (1966), 128-142.

4. KATRIŇ́́, T. Charakterisierung der Verallgemeinerten Stoneschen Halbverbande, Mat. Casop. 19 (1969), 235-247.

5. KATRIÑÁK, T. Pseudokomplementare Halbverbande, Mat. Casop. 1.8 (1968), 121-143.

6. VARLET, J.C. A generalization of the notion of pseudocomplementedness, Bul1 Soc. Roy. Sci. Liege 36 (1968), 149-158.

7. RHODES, J.B. Modular and distributive semilattices. Trans. Amer. Math. Soc., Vo1. 201 (1975), pp 31-41.

8. АввотT, J.C. Semi-Boolean algebra, Mat. Vestnik 4 (19) (1967), 177-198.

9. SPEED, T.P. A Note on commutative semigroups, J.Aust. Math. Soc., Vo1. VIII (1968), 731-736.

10. BIRKHOFF, G. Lattice Theory, American Mathematical Society, Colloquium Publications (Third edition 1967).

11. CORNISH, W.H. Pseudocomplemented modular semilattices, J. Aust. Math. Soc. Vo1. XVIII, (1974), 239-251. 


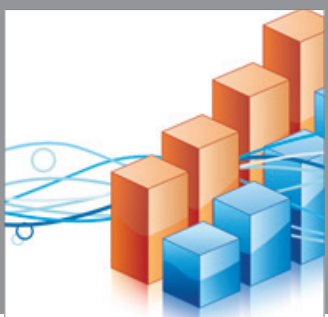

Advances in

Operations Research

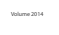

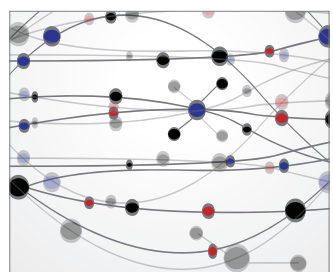

\section{The Scientific} World Journal
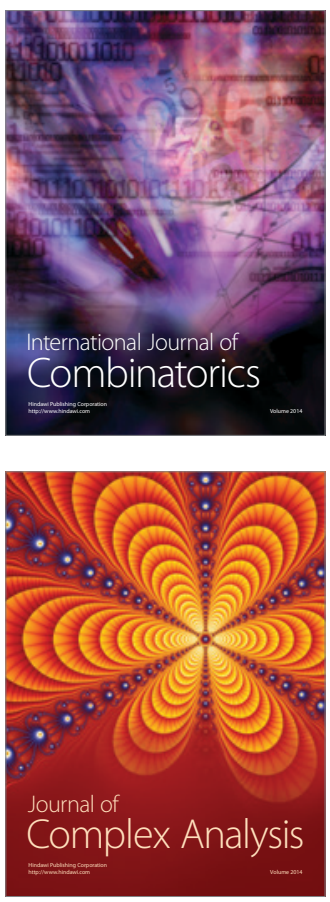

International Journal of

Mathematics and

Mathematical

Sciences
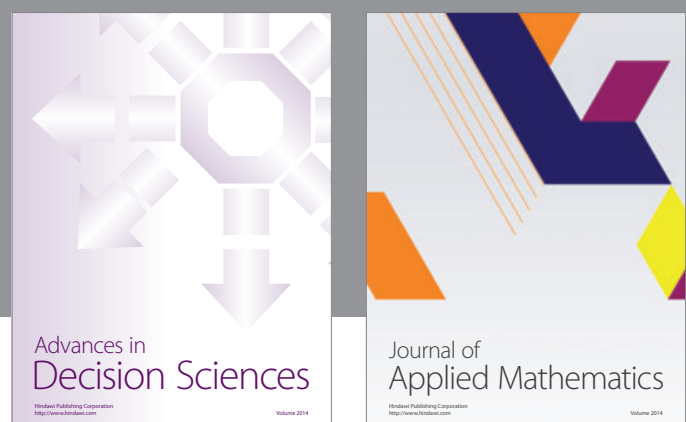

Journal of

Applied Mathematics
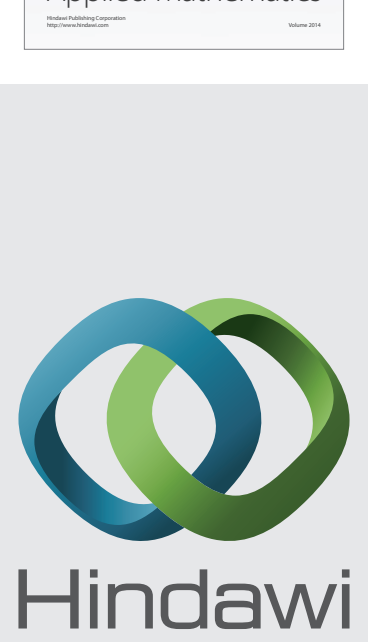

Submit your manuscripts at http://www.hindawi.com
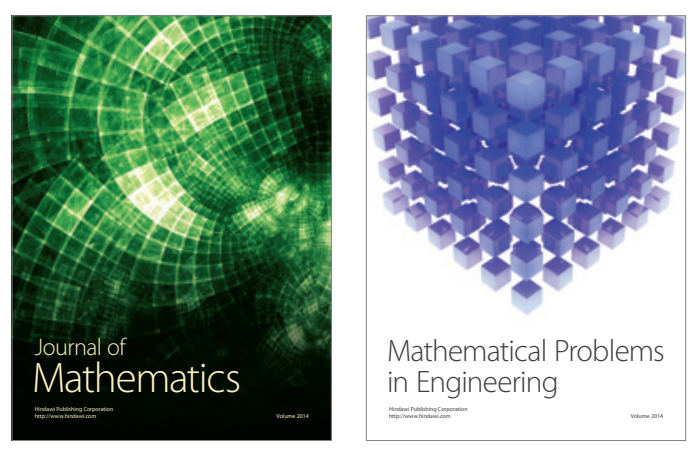

Mathematical Problems in Engineering
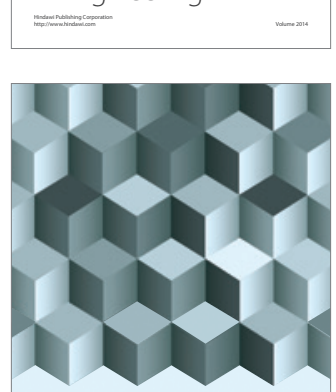

Journal of

Function Spaces
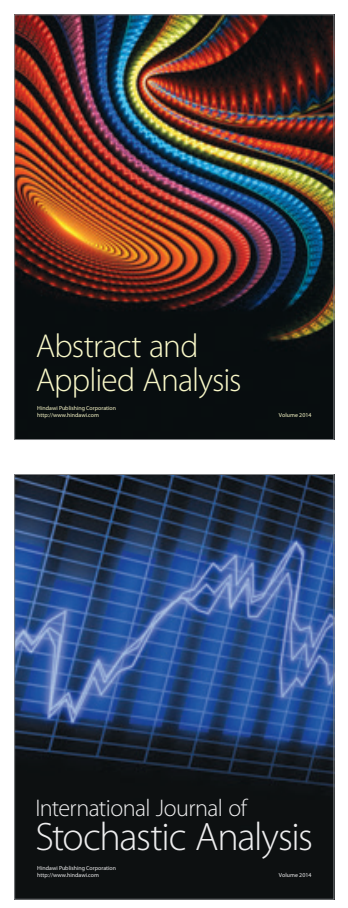

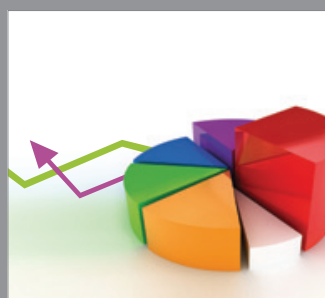

ournal of

Probability and Statistics

Promensencen
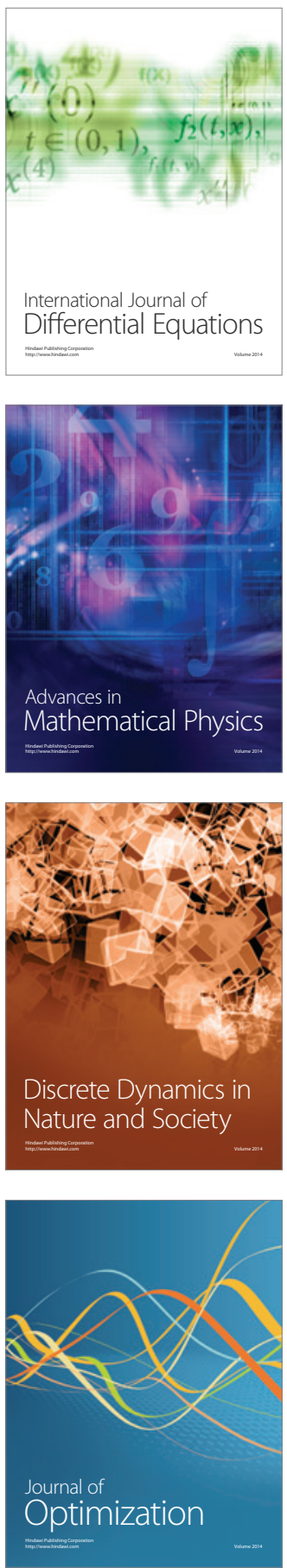